\title{
Cardiac valve calcification is associated with mortality in hemodialysis patients: a retrospective cohort study
}

\author{
Jiuxu Bai ${ }^{1 \dagger}$, Xiaoling Zhang ${ }^{2 \dagger}$, Aihong Zhang ${ }^{1}$, Yanping Zhang ${ }^{1}$, Kaiming Ren ${ }^{1}$, Zhuo Ren ${ }^{1}$, Chen Zhao , \\ Qian Wang ${ }^{1}$ and Ning Cao ${ }^{{ }^{*}}$
}

\begin{abstract}
Background: Cardiac valve calcification (CVC) is common in end-stage renal disease (ESRD). We investigated the effect of CVC on all-cause and cardiovascular (CV) mortality in maintenance hemodialysis (MHD) patients.

Methods: A retrospective cohort study was conducted on 434 hemodialysis patients who underwent echocardiography for qualitative assessment of valve calcification with complete follow-up data from January 1, 2014, to April 30, 2021. The baseline data between the CVC and non-CVC groups were compared. The Kaplan-Meier method was used to analyse all-cause and cardiovascular mortality. The association of CVC with all-cause and cardiovascular mortality was evaluated using multivariate Cox regression analysis.
\end{abstract}

Results: Overall, $27.2 \%$ of patients had mitral valve calcification (MVC), and 31.8\% had aortic valve calcification (AVC) on echocardiography. Patients with CVC showed significantly higher all-cause (log-rank $P<0.001)$ and cardiovascular (log-rank $P<0.001)$ mortality rates than patients without CVC. In multivariate regression analyses, MVC (HR: 1.517, $P=0.010$ ) and AVC (HR: 1.433, $P=0.028$ ) were significant factors associated with all-cause mortality. MVC (HR: 2.340, $P<0.001$ ) and AVC (HR: 2.410, $P<0.001$ ) were also significant factors associated with cardiovascular mortality.

Conclusions: MVC and AVC increased the risk of all-cause and cardiovascular mortality in MHD patients. Regular follow-up with echocardiography could be a useful method for risk stratification in MHD patients.

Keywords: Cardiac valve calcification, Mortality, Hemodialysis

\section{Background}

End-stage renal disease (ESRD) substantially increases the risk of cardiovascular morbidity and mortality [1]. Cardiovascular disease (CVD) is the leading cause of death in maintenance dialysis (MHD) patients. Cardiovascular $(\mathrm{CV})$ mortality risk in patients receiving haemodialysis or peritoneal dialysis is observed to be 10 to 20 times that of the general population [2]. Cardiac valve

\footnotetext{
*Correspondence: szxyjh@aliyun.com

${ }^{\dagger}$ Jiuxu Bai and Xiaoling Zhang equally contributed as shared first.

${ }^{1}$ Department of Blood Purification, General Hospital of Northern Theater

Command, 83 Wen Hua Road, Shenyang 110016, Liaoning, China

Full list of author information is available at the end of the article
}

calcification (CVC) plays an important role in cardiac structural changes and the progression to CVD and is frequently seen in patients undergoing dialysis. CVC, including aortic and mitral valve calcification (AVC and MVC), has been reported to occur with a higher prevalence (57.5\%) among incident hemodialysis patients [3]. Thus, derangements in calcium-phosphorous metabolism may contribute to CVC.

The association of cardiac valve (mitral and aortic) calcification and clinical outcomes has been studied in patients with end-stage renal disease (ESRD). CVC is correlated with higher cardiovascular and all-cause mortality risk in dialysis patients [4]. To better understand 
the relationship between valvular calcification and allcause and CV mortality in MHD patients, we conducted a retrospective cohort study. In this study, we aimed to investigate the association of cardiac parameters, valvular calcification, serum biochemical parameters, and clinical data with risk of cardiovascular or all-cause mortality in MHD patients.

\section{Methods}

This was a retrospective cohort study on 434 MHD patients who received hemodialysis at the General Hospital of Northern Theater Command and were enrolled in the cohort. This study collected data from January 1 , 2014, through December 31, 2014, and follow-up data were collected until April 30, 2021. The patients met the following inclusion criteria: (1) age over 18 years; (2) use of hemodialysis for $\geq 6$ months; (3) patients received hemodialysis 3 times per week in 4-h sessions using a dialysate calcium concentration of $1.5 \mathrm{mmol} / \mathrm{L}$; and (4) each patient had complete follow-up data, including echocardiographic parameters. The exclusion criteria were as follows: (1) patients who underwent kidney transplant or were changed to peritoneal dialysis; and (2) primary dilated cardiomyopathy. This study was approved by the Ethics Committee of General Hospital of Northern Theater Command.

All clinical data of MHD patients were collected and consisted of age, dialysis vintage, sex, primary disease, complications, medical history, height, weight, and smoking status. Phosphorus binder medication included calcium-based phosphate binders (calcium carbonate and calcium acetate) and calcium-free phosphate binders (lanthanum and sevelamer). Vitamin D medication included calcitriol and alfacalcidol. Nutritional vitamin D was not included in the study. Vitamin D, including calcitriol or alfacalcidol, was started at a low dose, independent of the initial PTH concentration, and then titrated based on the PTH response. Vitamin D was stopped when calcium $>2.5 \mathrm{mmol} / \mathrm{L}$. The intact PTH (iPTH) level was maintained in the range of approximately 2-9 times the upper normal limit for the assay based on the Kidney Disease Improving Global Outcomes (KDIGO) guidelines $[5,6]$. The body mass index (BMI) was measured as kilograms divided by the square of height in metres. Blood data were analysed from fasting blood samples obtained from a vein between 7:00 AM and 8:00 AM on the dialysis day within 3 months of the date of ultrasonic echocardiography. Blood tests were performed in the same laboratory using standard laboratory procedures and included haemoglobin (HGB), serum phosphate $(\mathrm{P})$, serum calcium (Ca), albumin (ALB), $\beta 2$-microglobulin, uric acid, iPTH and 25-hydroxy vitamin D (25(OH)D).
All echocardiographic examinations were performed at our hospital by experienced cardiologists on a nondialysis day. CVC was assessed by colour Doppler echocardiography, and other heart data were obtained. Valve calcification was defined as the presence of bright echoes $>1 \mathrm{~mm}$ on one or more cusps of the aortic valve, mitral valve, or mitral annulus [7]. The LV ejection fraction (LV-EF) was measured based on changes in the LV diameter or two-dimensional area between systole and diastole [8]. The left atrial dimension (LAD), left ventricular end-diastolic internal dimension (LVDd), left ventricular posterior wall thickness (LVPWT), and interventricular septal wall thickness (IVST) were obtained from M-mode tracings. The left ventricular mass index (LVMI) was also assessed. The left ventricular mass (LVM) was calculated using the following formula [echocardiographic assessment of left ventricular hypertrophy (LVH): comparison to necropsy findings]: $\mathrm{LVM}=(1.04 \times(\mathrm{IVST}+\mathrm{LVDd}$ + LVPWT) $\left.)^{3}-\operatorname{LVDd}^{3}\right) \times 0.8+0.6$ [9]. The LVMI was obtained by calculating the ratio of the LVM to the body surface area (BSA). The BSA formula for Chinese individuals is $\mathrm{S}=0.0061 \times$ height $+0.0124 \times \mathrm{W}-0.0099(\mathrm{~S}, \mathrm{BSA}$, $\mathrm{m} 2 ; \mathrm{H}$, height, $\mathrm{cm}$; W, weight, $\mathrm{kg}$ ) [10]. LVH was defined as LVMI $>115 \mathrm{~g} / \mathrm{m}^{2}$ (men) and $>95 \mathrm{~g} / \mathrm{m}^{2}$ (women) [11].

The endpoint events were all-cause death and cardiovascular death. Cardiovascular death was defined as myocardial infarction, heart failure, arrhythmia, sudden death, stroke, and other CV causes of death. Data for end points were obtained from hospital charts and through telephone interviews with patients conducted by trained reviewers who were blinded to the ultrasonic echocardiography analysis.

Data are expressed as the mean \pm SD or frequency (as percentage). Univariate survival analysis was performed with Kaplan-Meier analysis, and the overall significance was calculated by the log-rank test. Comparisons between groups were performed by an unpaired $t$-test or the nonparametric Wilcoxon rank-sum test in the case of nonnormally distributed variables. Continuous variables were compared between the groups using Student's $t$ test or the Mann-Whitney U-test, as appropriate. Categorical data were compared between the groups by the chi-square test. The Cox proportional hazards model was used to estimate the relative risk of all-cause mortality and cardiovascular mortality for different variables. All baseline variables with $P<0.05$ by univariate analysis were entered into a multivariate model to identify independent predictors for the end point. All statistical tests were performed at a two-sided significance level of 0.05 . Statistical Package for the Social Sciences (SPSS for Windows, IBM Corp, USA) version 22.0 was used for data analysis. 


\section{Results}

During a mean follow-up period of 70 months, 187 (43.1\% of total) patients died (135 of these from CVD causes).
Kaplan-Meier analyses were performed to examine the univariate association between the presence of $\mathrm{CVC}$ and outcome. Figures 1 and 2 show the relationship between

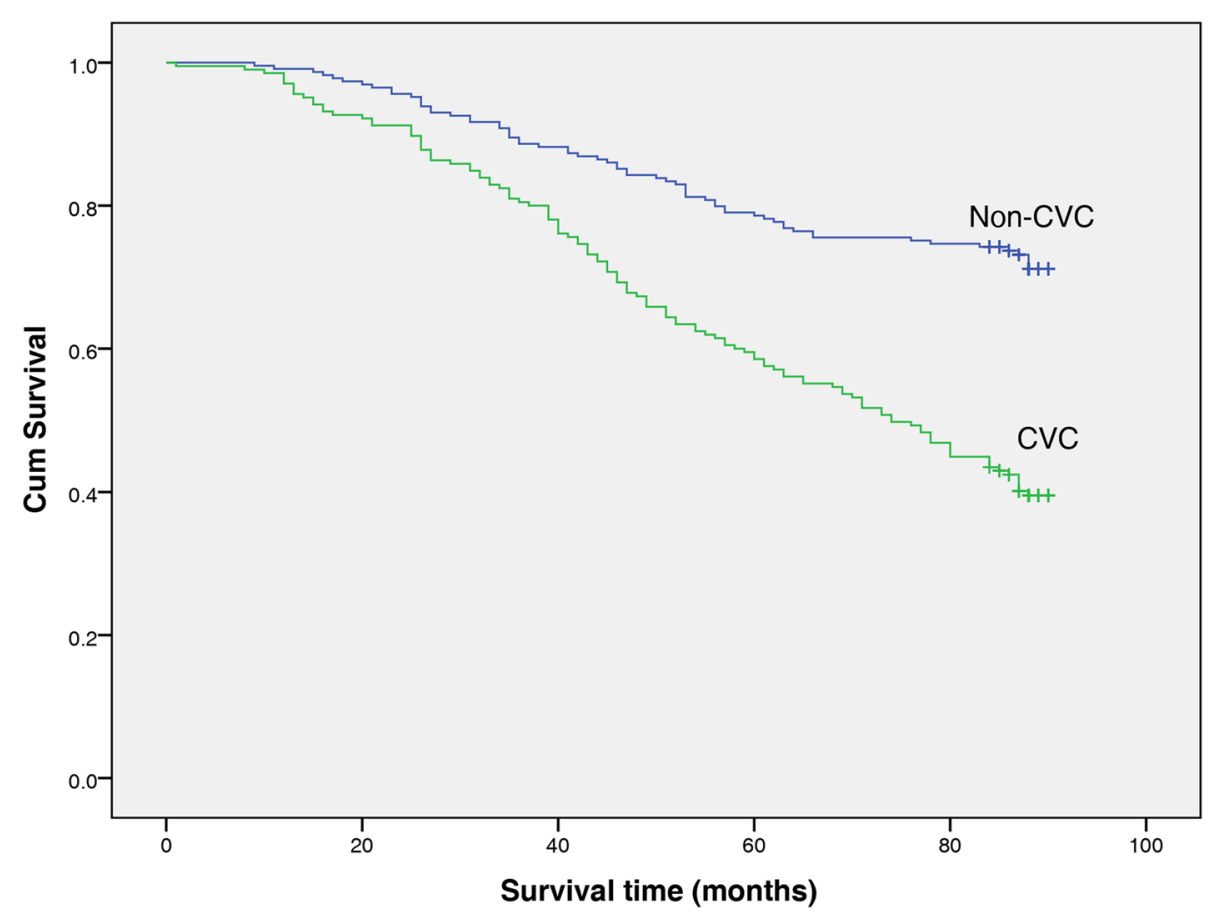

Fig. 1 Kaplan-Meier graph of all-cause mortality risk in the non-CVC and CVC groups

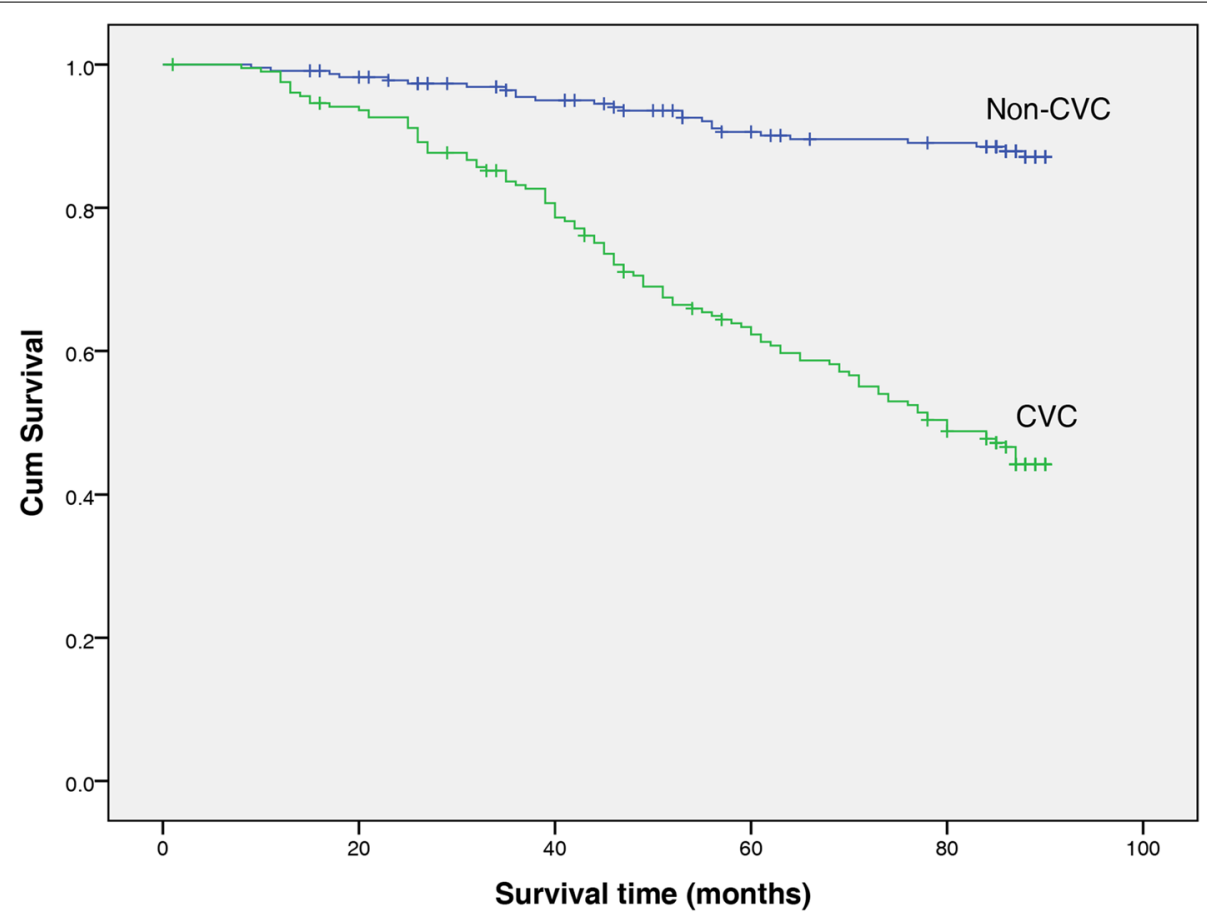

Fig. 2 Kaplan-Meier graph of cardiovascular mortality risk in the non-CVC and CVC groups 
CVC and death from all causes and CV mortality. The all-cause mortality rate was significantly higher among patients with CVC than non-CVC patients (log-rank test, $P<0.001)$. Similarly, the rate of CV mortality was significantly higher among patients with $\mathrm{CVC}$ than non-CVC patients $(\log$ rank test, $\mathrm{P}<0.001)$.

A total of 434 MHD patients were enrolled, consisting of $62.2 \%$ men and $37.8 \%$ women. The mean age was $53.08 \pm 13.39$ years. The characteristics of the study population are listed in Table 1. CVC (47.2\%) was observed in 434 patients: 118 (27.2\%) had MVC, 138 (31.8\%) had AVC, and 51 (11.8\%) had both MVC and AVC. Compared to non-CVC patients, patients with CVC were older and had more comorbidities. They were less often users of vitamin D medications. Moreover, they had a higher LAD, IVST, and LVMI. Conversely, the serum albumin level was lower in patients with valve calcification than in those without CVC.

The univariate analyses and Cox proportional hazards models are shown in Table 2. In the univariable analysis, CVC, MVC, AVC, age, diabetes history, CVD history, use of phosphorus-binding medications, use of vitamin D medications, LAD, LVMI, EF, HGB, ALB, $\beta 2$-microglobulin and iPTH were associated with allcause mortality (Table 2). In the univariable analysis, CVC, MVC, AVC, age, diabetes history, CVD history, use of phosphorus-binding medications, use of vitamin D medications, LAD, LVMI, EF, HGB, ALB and $\beta 2$-microglobulin were associated with $\mathrm{CV}$ mortality (Table 2).

Considering that MVC and AVC are the main manifestations of CVC in MHD patients, multivariate Cox proportional hazards analyses were conducted to identify factors associated with mortality. The presence of MVC was a significant factor associated with all-cause mortality (HR: $1.517, P=0.010$ ), in addition to old age, diabetes history, CVD history, no use of vitamin $\mathrm{D}$ medications, increased LAD, decreased HGB, increased $\beta 2$-microglobulin and decreased ALB (Table 3). The presence of AVC was a significant factor associated with all-cause mortality (HR: 1.433, $P=0.028$ in addition to old age, diabetes history, CVD history, no use of vitamin $\mathrm{D}$ medications, increased LAD, decreased HGB, increased $\beta 2$-microglobulin and decreased ALB.

A Cox proportional hazards regression analysis of risk factors for $\mathrm{CV}$ mortality is shown in Table 4 . After multivariate analysis, MVC (HR: $2.340, P<0.001$ ), diabetes history (HR: 2.538, $P<0.001$ ), CVD history (HR: 2.284, $P<0.001$ ), no use of vitamin D medications (HR: $0.579, P=0.007$ ), increased LAD (HR: $1.977, P=0.001$ ) and decreased HGB (HR: $0.987, P=0.018$ ) were associated with increased CV mortality. Moreover, AVC
(HR: 2.410, $P<0.001$ ), diabetes history (HR: 2.195, $P<0.001$ ), CVD history (HR: 2.141, $P<0.001$ ), no use of vitamin D medications (HR: 0.602, $P=0.013$ ), increased LAD (HR: 2.157, $P=0.001$ ) and decreased HGB (HR: $0.986, P=0.016$ ) were associated with increased CV mortality.

\section{Discussion}

In the present study, nearly half of MHD patients had MVC and/or AVC. The presence of MVC and/or AVC was associated with poor survival in this population, and both MVC and AVC were independently associated with an increased risk of all-cause mortality and CV mortality.

CVC screening of dialysis patients is performed with various imaging techniques, such as plain X-ray radiography, computed tomography (CT) and echocardiography. The use of CT for the diagnosis of MVC and AVC is highly reliable and sensitive but has several disadvantages, such as radiation exposure and high cost [12]. The echocardiographic evaluation of CVC is an easy, reproducible, noninvasive, and inexpensive method and is recommended as a routine examination for $\mathrm{CVC}$ screening by the KDIGO guidelines [13].

Many studies have demonstrated the predictive value of valve calcification for mortality; however, some controversies remain. A cohort study in Japan suggested that the presence of CVC could predict cardiovascular and all-cause mortality in incident hemodialysis patients [3]. MVC and AVC were associated with a significantly increased risk for all-cause mortality in 144 hemodialysis patients, who were followed for a median of 5.6years. However, after adjustment for multiple factors, MVC remained associated with all-cause mortality, whereas AVC was not [14]. A retrospective cohort study among 183 long-term hemodialysis patients showed that AVC increased the risk of cardiovascular death, whereas MVC did not [15]. Our study demonstrated that after adjusting for multiple factors, both MVC and AVC increased the risk of all-cause and CV mortality. The reasons for this finding may be the type of valve dysfunction that calcification causes. MVC may lead to mitral regurgitation, stenosis or both, whereas AVC can more often cause aortic stenosis. MVC has been directly related to an increased risk of CVD events, including coronary artery disease, acute myocardial infarction, stroke, and vascular diseases [16]. Aortic sclerosis was associated with a $50 \%$ higher risk of cardiovascular mortality and a $42 \%$ increased risk of myocardial infarction in the elderly [17]. Our findings may have important clinical implications. CVC on routine echocardiography should not be overlooked and can be used for risk assessment in MHD patients. Moreover, it can help identify patients who are appropriate for 
Table 1 Baseline clinical and lab characteristics in the CVC and non-CVC groups

\begin{tabular}{|c|c|c|c|}
\hline & Non-CVC (229) & CVC (205) & $P$ \\
\hline Age, (years) & $49.74 \pm 13.87$ & $56.81 \pm 11.78$ & $<0.001$ \\
\hline Duration of HD, (years) & $3.29 \pm 3.33$ & $3.58 \pm 3.19$ & 0.368 \\
\hline Men, n (\%) & $144(62.88 \%)$ & $126(61.46 \%)$ & 0.761 \\
\hline Smoking, n (\%) & $37(16.16 \%)$ & $26(12.68 \%)$ & 0.305 \\
\hline Hypertension history, n (\%) & $188(82.10 \%)$ & $172(83.90 \%)$ & 0.617 \\
\hline Diabetes, n (\%) & $44(19.21 \%)$ & $58(28.29 \%)$ & 0.026 \\
\hline Cardiovascular disease, $\mathrm{n}(\%)$ & 103 (44.98\%) & $137(66.83 \%)$ & $<0.001$ \\
\hline Phosphorus-binding medications, n (\%) & $179(78.17 \%)$ & $147(71.71 \%)$ & 0.12 \\
\hline Vitamin D medications, n (\%) & $198(86.46 \%)$ & $159(77.56 \%)$ & 0.015 \\
\hline $\mathrm{BMI}\left(\mathrm{kg} / \mathrm{m}^{2}\right)$ & $22.98 \pm 3.85$ & $22.54 \pm 3.72$ & 0.179 \\
\hline $\operatorname{LAD}(\mathrm{cm})$ & $3.38 \pm 0.50$ & $3.60 \pm 0.55$ & $<0.001$ \\
\hline LVDd (cm) & $4.74 \pm 0.61$ & $4.77 \pm 0.73$ & 0.94 \\
\hline IVST (cm) & $1.19 \pm 0.13$ & $1.25 \pm 0.17$ & $<0.001$ \\
\hline LVPWT (cm) & $1.14 \pm 0.13$ & $1.15 \pm 0.13$ & 0.254 \\
\hline $\operatorname{LVMI}\left(\mathrm{g} / \mathrm{m}^{2}\right)$ & $119.38 \pm 34.04$ & $128.07 \pm 38.10$ & 0.013 \\
\hline $\mathrm{EF}$ & $0.62 \pm 0.08$ & $0.61 \pm 0.11$ & 0.678 \\
\hline $\mathrm{HGB}(\mathrm{g} / \mathrm{L})$ & $105.15 \pm 15.24$ & $102.77 \pm 16.82$ & 0.122 \\
\hline $\mathrm{Ca}(\mathrm{mmol} / \mathrm{L})$ & $2.22 \pm 0.23$ & $2.24 \pm 0.24$ & 0.438 \\
\hline $\mathrm{P}(\mathrm{mmol} / \mathrm{L})$ & $2.09 \pm 0.62$ & $2.06 \pm 0.62$ & 0.558 \\
\hline ß2-Microglobulin (mg/L) & $29.29 \pm 6.96$ & $30.25 \pm 7.25$ & 0.158 \\
\hline iPTH (pg/mL) & $389.75 \pm 387.76$ & $363.69 \pm 382.43$ & 0.379 \\
\hline ALB (g/L) & $40.30 \pm 3.37$ & $39.34 \pm 3.43$ & 0.004 \\
\hline $\mathrm{UA}(\mu \mathrm{mol} / \mathrm{L})$ & $473.75 \pm 96.25$ & $460.57 \pm 95.41$ & 0.154 \\
\hline $25(\mathrm{OH}) \mathrm{D}(\mathrm{ng} / \mathrm{mL})$ & $19.81 \pm 10.76$ & $19.45 \pm 9.52$ & 0.714 \\
\hline
\end{tabular}

intensive medical treatment to reduce cardiovascular events.

In our study, we also focused on the relationship between the measured cardiac parameters and all-cause and cardiovascular death in MHD patients. LVMI was an independent risk factor in univariate Cox regression, but it was not significant in multivariate Cox regression after adjusting for other factors. However, LVMI is frequently reported as an independent mortality predictor among maintenance hemodialysis patients [18]. Accordingly, in other cohort studies, the progression of LVH was significantly related to all-cause mortality and cardiovascular events in 161 hemodialysis patients [19]. Our data show that LAD is significantly associated with all-cause and CV mortality, which is consistent with the results of previous studies. A study performed by Milan D et al. showed that a large left atrial diameter was an independent predictor of mortality in hemodialysis patients or renal allograft recipients [20, 21]. The left atrial dimensions greatly depend on the left ventricular pressure. When the ventricle is exposed to pressure and/or volume overload, such as in heart failure, hypertension or valve disease, this mechanism results in progressive left atrial enlargement. Left atrial dysfunction has been observed in association with mitral stenosis [22]. Left atrial pressure and volume overload provoke functional alterations in the atrial myocardium in mitral stenosis and correlate with exercise capacity in other causes of heart failure, which promote cardiac myocyte overstretching [23]. Mitral stenosis can cause left atrial enlargement from pressure or volume overload. A prospective study involving 249 patients with ESRD reported that dialysis patients who died during the follow-up had a higher LAD than survivors [24]. Thus, an increased LAD may signify increased cardiovascular risk in patients with CKD.

One of the other important findings of this study is that MHD patients who received oral active vitamin D treatment had significantly lower risks of cardiovascular and all-cause mortality. Experimental models suggest that activated vitamin D attenuates the development of LVH, improves left ventricular diastolic function, and reduces episodes of heart failure [25, 26]. In observational studies, MHD patients with a higher mortality risk may not have received vitamin $\mathrm{D}$ analogues, leading to an apparent association between vitamin $\mathrm{D}$ administration and better survival [27]. In this historical cohort study, chronic hemodialysis patients in the group that received injectable vitamin D showed a significant survival advantage 
Table 2 Univariate Cox analysis of factors in relation to all-cause and cardiovascular mortality

\begin{tabular}{|c|c|c|c|c|}
\hline \multirow[t]{2}{*}{ Variable } & \multicolumn{2}{|l|}{ All-cause mortality } & \multicolumn{2}{|c|}{ Cardiovascular mortality } \\
\hline & HR(95\%) & $P$ & HR(95\%) & $P$ \\
\hline CVC & $2.647(1.955-3.583)$ & $<0.001$ & 5.992(3.877-9.261) & $<0.001$ \\
\hline MVC & $1.927(1.434-2.589)$ & $<0.001$ & $3.161(2.251-4.439)$ & $<0.001$ \\
\hline AVC & $2.650(1.988-3.533)$ & $<0.001$ & $4.177(2.951-5.912)$ & $<0.001$ \\
\hline Age & $1.047(1.035-1.058)$ & $<0.001$ & $1.039(1.025-1.025)$ & $<0.001$ \\
\hline Duration of HD & $0.971(0.928-1.017)$ & 0.214 & $0.967(0.916-1.022)$ & 0.231 \\
\hline Sex (male vs female) & $0.922(0.687-1.238)$ & 0.588 & $0.922(0.687-1.238)$ & 0.893 \\
\hline Smoking & $1.081(0.724-1.616)$ & 0.703 & $1.267(0.808-1.985)$ & 0.302 \\
\hline Hypertension history & $1.162(0.782-1.727)$ & 0.456 & $1.474(0.886-2.452)$ & 0.135 \\
\hline Diabetes & $2.797(2.078-3.765)$ & $<0.001$ & $2.984(2.106-4.228)$ & $<0.001$ \\
\hline CVD & $1.713(1.268-2.315)$ & $<0.001$ & $2.571(1.748-3.780)$ & $<0.001$ \\
\hline Phosphorus-binding medications & $0.492(0.354-0.683)$ & $<0.001$ & $0.588(0.392-0.883)$ & 0.011 \\
\hline Vitamin D medications & $0.427(0.317-0.576)$ & $<0.001$ & $0.437(0.306-0.622)$ & $<0.001$ \\
\hline BMI & $1.003(0.966-1.043)$ & 0.859 & $1.008(0.964-1.054)$ & 0.734 \\
\hline LAD & $1.817(1.419-2.328)$ & $<0.001$ & $2.292(1.729-3.037)$ & $<0.001$ \\
\hline LVMI & $1.659(1.204-2.284)$ & 0.002 & $1.890(1.281-2.790)$ & 0.001 \\
\hline $\mathrm{EF}$ & $0.197(0.043-0.912)$ & 0.038 & $0.128(0.022-0.740)$ & 0.022 \\
\hline$H G B$ & $0.984(0.975-0.993)$ & $<0.001$ & $0.982(0.972-0.993)$ & 0.001 \\
\hline ß2-Microglobulin & $1.032(1.011-1.054)$ & 0.003 & $1.026(1.001-1.052)$ & 0.038 \\
\hline ALB & $0.881(0.847-0.917)$ & $<0.001$ & $0.915(0.872-0.961)$ & $<0.001$ \\
\hline UA & 0.999(0.997-1.000) & 0.095 & 0.998(0.997-1.000) & 0.099 \\
\hline $25(\mathrm{OH}) \mathrm{D}$ & 0.993(0.978-1.008) & 0.338 & 0.992(0.974-1.009) & 0.356 \\
\hline \multicolumn{5}{|l|}{ iPTH $150-300 \mathrm{pg} / \mathrm{mL}$ (as reference) } \\
\hline iPTH $<150 \mathrm{pg} / \mathrm{mL}$ & $1.516(1.033-2.226)$ & 0.034 & $1.327(0.84-2.098)$ & 0.225 \\
\hline iPTH $>300 \mathrm{pg} / \mathrm{mL}$ & $1.081(0.741-1.575)$ & 0.687 & $1.107(0.715-1.712)$ & 0.687 \\
\hline \multicolumn{5}{|l|}{ Ca $2.1-2.5 \mathrm{mmol} / \mathrm{L}$ (as reference) } \\
\hline $\mathrm{Ca}<2.1 \mathrm{mmol} / \mathrm{L}$ & $1.091(0.792-1.504)$ & 0.593 & $1.144(0.781-1.676)$ & 0.488 \\
\hline $\mathrm{Ca}>2.5 \mathrm{mmol} / \mathrm{L}$ & 0.954(0.603-1.508) & 0.839 & 1.194(0.721-1.977) & 0.490 \\
\hline \multicolumn{5}{|l|}{ P 1.13-1.78 mmol/L (as reference) } \\
\hline $\mathrm{P}<1.13 \mathrm{mmol} / \mathrm{L}$ & $1.24(0.593-2.591)$ & 0.567 & $0.645(0.2-2.079)$ & 0.463 \\
\hline $\mathrm{P}>1.78 \mathrm{mmol} / \mathrm{L}$ & $0.897(0.659-1.222)$ & 0.492 & $0.920(0.640-1.322)$ & 0.653 \\
\hline
\end{tabular}

over patients who did not [28]. However, the PRIMO and OPERA studies failed to demonstrate improvements in clinically relevant outcomes but demonstrated an increased risk of hypercalcaemia in CKD $[29,30]$. The participants in the PRIMO and OPERA trials only had moderately increased PTH levels. In addition, active vitamin D will increase the intestinal calcium and phosphate absorption, which will worsen the vascular calcification. Thus, for severe and progressive secondary hyperparathyroidism (SHPT) in CKD G5D, calcitriol or vitamin $\mathrm{D}$ analogues should be considered, with an initially low dose, independent of the initial PTH concentration, and then titrated based on the PTH response, and hypercalcaemia should be avoided [6]. An observational study of chronic hemodialysis patients suggested a decrease in $\mathrm{CV}$ mortality among patients who received vitamin $\mathrm{D}$ to confirm the possibility that such medication improves the survival rate of ESRD patients [31]. We believe that our findings may have important clinical implications when vitamin $\mathrm{D}$ supplementation is considered a relatively easy, safe and inexpensive therapy in MHD patients suffering from SHPT. Further randomized and controlled studies are necessary to confirm the beneficial effect of vitamin D in lowering the cardiovascular mortality risk in patients with ESRD.

The major limitations of this study are its retrospective, single-centre design and a possible selection bias of excluding some patients without echocardiography or other examination data. Another limitation of this study is that cardiac ultrasound results were collected to evaluate whether there was valve calcification at the time of enrolment. However, the progression of CVC was not followed up. The strengths of our study include the relatively large number of participants, the long follow-up 
Table 3 Multivariate Cox regression models for all-cause mortality

\begin{tabular}{|c|c|c|c|c|}
\hline \multirow[t]{2}{*}{ Variable } & \multicolumn{2}{|l|}{ MVC } & \multicolumn{2}{|l|}{ AVC } \\
\hline & HR(95\%) & $P$ & $\mathrm{HR}(95 \%)$ & $P$ \\
\hline mitral or aortic valve calcification & $1.517(1.105-2.082)$ & 0.010 & 1.433(1.039-1.976) & 0.028 \\
\hline Age & $1.028(1.015-1.041)$ & $<0.001$ & $1.026(1.013-1.039)$ & $<0.001$ \\
\hline Diabetes & $2.157(1.573-2.957)$ & $<0.001$ & $2.023(1.475-2.773)$ & $<0.001$ \\
\hline CVD & $1.649(1.211-2.247)$ & 0.002 & $1.625(1.186-2.225)$ & 0.002 \\
\hline Phosphorus-binding medications & $0.736(0.506-1.069)$ & 0.107 & $0.773(0.528-1.132)$ & 0.186 \\
\hline Vitamin D medications & $0.736(0.506-1.069)$ & 0.036 & $0.702(0.496-0.994)$ & 0.046 \\
\hline LAD & $1.506(1.067-2.125)$ & 0.020 & $1.562(1.104-2.209)$ & 0.012 \\
\hline LVMI & 0.999(0.994-1.004) & 0.616 & $0.998(0.993-1.004)$ & 0.569 \\
\hline $\mathrm{EF}$ & $0.639(0.108-3.771)$ & 0.621 & $0.657(0.107-4.017)$ & 0.649 \\
\hline HGB & 0.988(0.979-0.997) & 0.011 & $0.988(0.979-0.997)$ & 0.011 \\
\hline ß2-Microglobulin & $1.022(1.000-1.045)$ & 0.046 & $1.030(1.008-1.053)$ & 0.007 \\
\hline ALB & $0.942(0.901-0.985)$ & 0.009 & $0.947(0.905-0.990)$ & 0.015 \\
\hline \multicolumn{5}{|l|}{ iPTH 150-300 pg/mL (as reference) } \\
\hline iPTH $<150 \mathrm{pg} / \mathrm{mL}$ & $1.317(0.873-1.987)$ & 0.189 & $1.240(0.822-1.870)$ & 0.304 \\
\hline iPTH > 300 pg/mL & $1.371(0.925-2.032)$ & 0.116 & $1.289(0.877-1.912)$ & 0.206 \\
\hline
\end{tabular}

Table 4 Multivariate Cox regression models for cardiovascular mortality

\begin{tabular}{|c|c|c|c|c|}
\hline \multirow[t]{2}{*}{ Variable } & \multicolumn{2}{|l|}{ MVC } & \multicolumn{2}{|l|}{ AVC } \\
\hline & HR(95\%) & $P$ & HR(95\%) & $P$ \\
\hline mitral or aortic valve calcification & $2.340(1.625-3.370)$ & $<0.001$ & $2.410(1.632-3.559)$ & $<0.001$ \\
\hline Age & $1.015(1.000-1.030)$ & 0.056 & $1.010(0.994-1.026)$ & 0.223 \\
\hline Diabetes & $2.538(1.747-3.688)$ & $<0.001$ & $2.195(1.507-3.197)$ & $<0.001$ \\
\hline CVD & $2.284(1.535-3.398)$ & $<0.001$ & $2.141(1.443-3.179)$ & $<0.001$ \\
\hline Phosphorus-binding medications & $0.847(0.543-1.319)$ & 0.462 & $0.952(0.602-1.505)$ & 0.832 \\
\hline Vitamin D medications & $0.579(0.389-0.864)$ & 0.007 & $0.602(0.403-0.899)$ & 0.013 \\
\hline LAD & $1.977(1.353-2.889)$ & 0.001 & $2.157(1.455-3.197)$ & 0.001 \\
\hline LVMI & $0.997(0.991-1.003)$ & 0.282 & $0.996(0.990-1.002)$ & 0.207 \\
\hline $\mathrm{EF}$ & $0.597(0.080-4.438)$ & 0.615 & $0.872(0.108-7.031)$ & 0.897 \\
\hline$H G B$ & $0.987(0.976-0.998)$ & 0.018 & $0.986(0.975-0.997)$ & 0.016 \\
\hline ß2-Microglobulin & $1.018(0.994-1.044)$ & 0.145 & $1.035(1.010-1.061)$ & 0.007 \\
\hline ALB & $0.978(0.928-1.032)$ & 0.420 & $0.990(0.940-1.042)$ & 0.691 \\
\hline
\end{tabular}

duration, and the ability to adjust for several established risk factors and potential confounders.

\section{Conclusions}

In summary, our study demonstrated that CVC, including $\mathrm{MVC}$ and $\mathrm{AVC}$, increases the risk of all-cause and cardiovascular mortality in MHD patients. Regular follow-up by echocardiography could be a useful method for risk stratification in MHD patients. Multicentre, large-sample, prospective cohort, follow-up studies are needed to confirm the reproducibility of our results and determine whether intervening in the progression of CVC can reduce the mortality rate among MHD patients.

\section{Abbreviations}

CVC: Cardiac valve calcification; ESRD: End stage renal disease; CV: Cardiovascular; MVC: Mitral valve calcification; AVC: Aortic valve calcification; CVD: Cardiovascular disease; iPTH: Intact parathyroid hormone; 25(OH)D: 25 hydroxy vitamin D; LV-EF: Left ventricular ejection fraction; LAD: Left atrial dimension; LVDd: Left ventricular end-diastolic internal dimension; LVPWT: Left ventricular posterior wall thickness; IVST: Interventricular septal wall thickness; LVMI: Left ventricular mass index; LVM: Left ventricular mass.

\section{Acknowledgements}

Not applicable. 


\section{Authors' contributions}

$J X B, X L Z$, and NC conceived the study and its design, had full access to the patient records, and take responsibility for the accuracy and integrity of the data. JXB, XLZ and AHZ contributed to analysis and interpretation of data. YPZ, $\mathrm{KMR}, \mathrm{ZR}, \mathrm{CZ}$, and QW participated in patient inclusion and demographic data collection. All authors critically revised the drafted manuscript. JXB and XLZ equally contributed as shared first, NC contributed as the senior author. The author(s) have read and approved the final manuscript.

\section{Funding}

This work was supported by the Natural Science Foundation of Liaoning Province (20180550009).

\section{Availability of data and materials}

Data that support the results in the article can be found by academic researchers by sending an email to the corresponding author at szxyjh@ aliyun.com.

\section{Declarations}

\section{Ethics approval and consent to participate}

This research has been approved by the Ethics Committee of General Hospital of Northern Theater Command. Informed consent was waived by the Ethics Committee of General Hospital of Northern Theater Command due to the retrospective nature of the study. All methods were performed in accordance with relevant guidelines and regulations.

\section{Consent for publication}

Not applicable.

\section{Competing interests}

The authors declare that they have no competing interests.

\section{Author details}

'Department of Blood Purification, General Hospital of Northern Theater Command, 83 Wen Hua Road, Shenyang 110016, Liaoning, China. ${ }^{2}$ Department of Nephrology, Jin Qiu Hospital of Liaoning Province (Geriatric Hospital of Liaoning Province), Shenyang, China.

Received: 25 September 2021 Accepted: 12 January 2022 Published online: 22 January 2022

\section{References}

1. Go AS, Chertow GM, Fan D, Mcculloch CE, Hsu CY. Chronic kidney disease and the risks of death, cardiovascular events, and hospitalization. N Engl J Med. 2004;41(13):177.

2. Foley R. Epidemiology of cardiovascular disease in chronic renal disease. J Am Soc Nephrol. 1998:9:S16-23.

3. Takahashi H, Ishii H, Aoyama T, Kamoi D, Kasuga H, Ito Y, et al. Association of Cardiac Valvular Calcifications and C-reactive protein with cardiovascular mortality in incident hemodialysis patients: a Japanese cohort study. Am J Kidney Dis. 2013;61(2):254-61.

4. Wang $Z$, Jiang $A$, Wei $F$, Chen $\mathrm{H}$. Cardiac valve calcification and risk of cardiovascular or all-cause mortality in dialysis patients: a meta-analysis. BMC Cardiovasc Disord. 2018;18(1):12.

5. Kidney Disease: Improving Global Outcomes CKDMBDWG. KDIGO clinical practice guideline for the diagnosis, evaluation, prevention, and treatment of chronic kidney Disease-mineral and bone disorder (CKD-MBD). Kidney Int Suppl. 2009;113:S1-130.

6. Kidney Disease: Improving Global Outcomes CKDMBDUWG. KDIGO 2017 clinical practice guideline update for the diagnosis, evaluation, prevention, and treatment of chronic kidney Disease-mineral and bone disorder (CKD-MBD). Kidney Int Suppl. 2017;7(1):1-59.

7. Wang AY, Wang M, Woo J, Lam CW, Li PK, Lui SF, et al. Cardiac valve calcification as an important predictor for all-cause mortality and cardiovascular mortality in long-term peritoneal dialysis patients: a prospective study. J Am Soc Nephrol. 2003;14(1):159-68.
8. Quinones MA, Pickering E, Alexander JK. Percentage of shortening of the echocardiographic left ventricular dimension. Its use in determining ejection fraction and stroke volume. Chest. 1978;74(1):59-65.

9. Lang RM, Bierig M, Devereux RB, Flachskampf FA, Foster E, Pellikka PA, et al. Recommendations for chamber quantification. Eur J Echocardiogr. 2006;7(2):79-108

10. Stevenson PH. Height.weight.surface formula for the estimation of surface area in Chinese subjects. Chin J Phys. 1937;12:327-30.

11. Mancia G, Fagard R, Narkiewicz K, Redon J, Zanchetti A, Boehm M, Christiaens T, Cifkova R, Backer GD, Dominiczak A. 2013 ESH/ESC guidelines for the management of arterial hypertension: the Task Force for the Management of Arterial Hypertension of the European Society of Hypertension (ESH) and of the European Society of Cardiology (ESC). J Hypertens. 2013:31(7):1281-357.

12. Hensen L, El MM, Van R, Smit JM, Wouter JJ, Bax JJ, et al. Prevalence and prognostic implications of mitral and aortic valve calcium in patients with chronic kidney Disease. Am J Cardiol. 2018;122:S0002914918316394.

13. Group KDIGOC-MW. KDIGO clinical practice guideline for the diagnosis, evaluation, prevention, and treatment of Chronic Kidney Disease-Mineral and Bone Disorder (CKD-MBD). Kidney Int Suppl. 2009;(113):S1-130.

14. Raggi P, Bellasi A, Gamboa C, Ferramosca E, Ratti C, Block GA, et al. Allcause mortality in hemodialysis patients with heart valve calcification. Clin J Am Soc Nephrol. 2011;6(8):1990-5.

15. Li M, Ye ZC, Li CM, Zhao WB, Lou TQ. The influence of cardiac valvular calcification on all-cause and cardiovascular mortality in maintenance hemodialysis patients. Int Urol Nephrol. 2020;52(6):943-51.

16. Fox, CS, Vasan RS, Parise H, Levy D, O'Donnell CJ, D'Agostino RB, Benjamin EJ, Framingham Heart Study: Mitral Annular Calcification Predicts Cardiovascular Morbidity and Mortality: the Framingham Heart Study. Circulation. 2003;107(11):1492-6.

17. Otto CM, Lind BK, Kitzman DW, Gersh BJ, Siscovick DS. Association of aortic-valve sclerosis with cardiovascular mortality and morbidity in the elderly. N Engl J Med. 1999;341(3):142-7.

18. Sha L, Zhang DL, Wang G, Cui WY, Liu WH. Left ventricular mass index and aortic arch calcification score are independent mortality predictors of maintenance hemodialysis patients. Hemodial Int. 2012;16(4):504-11.

19. Zoccali C, Benedetto FA, Mallamaci F, Tripepi G, Malatino LS. Left ventricular mass monitoring in the follow-up of dialysis patients: prognostic value of left ventricular hypertrophy progression. Kidney Int. 2004;65(4):1492-8.

20. Stosovic M, Petrovic MZ, Vujisic-Tesic BD, Stanojevic ML, Naumovic RT. Predictive value of echocardiography and its relation to Kt/ $\mathrm{V}$ and anthropometric parameters in hemodialysis patients. Ren Fail. 2015:37(4):1-8.

21. Kainz A, Goliasch G, Wiesbauer F, Binder T, Maurer G, Nesser HJ, et al. Left atrial diameter and survival among renal allograft recipients. Clin J Am Soc Nephrol. 2013;8(12):2100-5.

22. Karabay CY, Akgun T. The impact of mitral stenosis on left atrial function assessed by two-dimensional speckle tracking echocardiography. Echocardiography. 2013;30(7):863.

23. Trikas A, Papathanasiou S, Tousoulis D, Tentolouris K, Vasiliadou K, Antoniades $C$, et al. Left atrial function, cytokines and soluble apoptotic markers in mitral stenosis: effects of valvular replacement. Int J Cardiol. 2005;99(1):111-5

24. Tripepi G, Benedetto FA, Mallamaci F, Tripepi R, Malatino L, Zoccali C. Left atrial volume monitoring and cardiovascular risk in patients with end-stage renal disease: a prospective cohort study. J Am Soc Nephrol. 2007;18(4):1316-22.

25. Bodyak N, Ayus JC, Achinger S, Shivalingappa V, Ke Q, Chen YS, et al. Activated vitamin D attenuates left ventricular abnormalities induced by dietary sodium in Dahl salt-sensitive animals. Proc Natl Acad Sci U S A. 2007:104(43):16810-5.

26. Bae S, Yalamarti B, Ke Q, Choudhury S, Yu H, Karumanchi SA, et al. Preventing progression of cardiac hypertrophy and development of heart failure by paricalcitol therapy in rats. Cardiovasc Res. 2011;91(4):632-9.

27. Kalantar-Zadeh K, Kuwae N, Regidor DL, Kovesdy CP, Kilpatrick RD, Shinaberger CS, et al. Survival predictability of time-varying indicators of bone disease in maintenance hemodialysis patients. Kidney Int. 2006;70(4):771-80.

28. Teng M, Wolf M, Ofsthun MN, Lazarus JM, Hernan MA, Camargo CA Jr, et al. Activated injectable vitamin D and hemodialysis survival: a historical cohort study. J Am Soc Nephrol. 2005;16(4):1115-25. 
29. Thadhani R, Appelbaum E, Pritchett Y, Chang Y, Wenger J, Tamez H, et al. Vitamin D therapy and cardiac structure and function in patients with chronic kidney disease: the PRIMO randomized controlled trial. Jama. 2012;307(7):674-84.

30. Wang AY, Fang F, Chan J, Wen YY, Qing S, Chan IH, et al. Effect of paricalcitol on left ventricular mass and function in CKD--the OPERA trial. J Am Soc Nephrol. 2014;25(1):175-86.

31. Shoji T, Shinohara K, Kimoto E, Emoto M, Tahara H, Koyama H, et al. Lower risk for cardiovascular mortality in oral 1alpha-hydroxy vitamin D3 users in a haemodialysis population. Nephrol Dial Transplant. 2004;19(1):179-84.

\section{Publisher's Note}

Springer Nature remains neutral with regard to jurisdictional claims in published maps and institutional affiliations.

- fast, convenient online submission

- thorough peer review by experienced researchers in your field

- rapid publication on acceptance

- support for research data, including large and complex data types

- gold Open Access which fosters wider collaboration and increased citations

- maximum visibility for your research: over $100 \mathrm{M}$ website views per year

At BMC, research is always in progress.

Learn more biomedcentral.com/submissions 